Archives de sciences sociales des religions

124 | octobre - décembre 2003

Varia

\title{
Charles-Henry Pradelles de Latour, Incroyance et paternités
}

Paris, Epel, 2001, 232 p. (bibliogr., glossaire, index, tablx.)

Martine Gross

\section{(2) OpenEdition}

\section{Journals}

Édition électronique

URL : http://journals.openedition.org/assr/910

DOI : $10.4000 /$ assr. 910

ISSN : $1777-5825$

Éditeur

Éditions de l'EHESS

Édition imprimée

Date de publication : 1 octobre 2003

Pagination : 63-170

ISBN : 2-222-96739-2

ISSN : 0335-5985

\section{Référence électronique}

Martine Gross, «Charles-Henry Pradelles de Latour, Incroyance et paternités », Archives de sciences sociales des religions [En ligne], 124 | octobre - décembre 2003, document 124.45, mis en ligne le 25 octobre 2005, consulté le 24 septembre 2020. URL : http://journals.openedition.org/assr/910 ; DOI https://doi.org/10.4000/assr.910 
sous les formes les plus contradictoires, les plus inattendues... L'Église catholique a passé son temps à gérer la diversité... La diversité ne fait pas difficulté, c'est aussi un envers de l'histoire religieuse contemporaine" (pp. 203-204).

En somme, et si nous avons bien compris, sans doute le christianisme catholique est en mauvaise posture. Mais pourquoi devrait-il mourir? C'est l'espérance d'É.P. qu'en reprenant la tradition populaire qui passe par François d'Assise, Vincent de Paul, et toute une postérité encore vivante parmi nous en Europe et ailleurs, moyennant aussi une conversion intellectuelle et spirituelle sérieuse, il a encore sa chance. Et l'historien ne pense pas que l'administration de l'Église s'opposerait à ce projet: «Le Saint Office n'intervient que quand il y a une histoire, des difficultés, mais le plus souvent la diversité ne fait pas difficulté, c'est aussi un envers de l'histoire religieuse contemporaine ».

Contrairement à d'autres observateurs de la conjoncture ecclésiastique et sociale, É.P. ne pense pas que l'histoire doive se clore. L'histoire est toujours ouverte au renouvellement tant qu'il y a des hommes pour en être les acteurs. La réussite n'est jamais garantie à aucune entreprise; celles de 2000 ans d'âge meurent plus lentement et difficilement que d'autres. S'il faut voter, É.P. vote pour l'espérance.

\section{Jean Séguy.}

Incroyance et paternités. Paris, Epel, 2001, 232 p. (bibliogr., glossaire, index, tablx.).

L'auteur introduit les croyances dans l'étude des systèmes de parenté et montre qu'elles étayent les différents types de filiation, patri- et matrilinéaire et plus particulièrement leurs conceptions de la paternité. Cette approche fait ressortir la place occupée par l'incroyance qui sous-tend de différentes manières, les systèmes d'alliance matrimoniale.

Lorsqu'une femme quitte sa lignée pour rejoindre son mari, elle devient épouse et mère des enfants. Le père paie pour acquérir une femme, le prix de la fiancée. Chez les patrilinéaires, la sexualité est associée très étroitement à la procréation. Le prix payé donne au mari un droit sur les enfants à naître. Si une femme s'avère stérile, le mari peut en prendre une autre. L'alliance est fondée sur la filiation. L'A. s'appuie sur les données ethnographiques recueillies sur le système patrilinéaire des
Bamilékés du Cameroun et sur le système matrilinéaire des Trobriandais de Papouasie Nouvelle-Guinée.

Chez les matrilinéaires, les enfants, quels que soient les géniteurs, appartiennent au groupe de leur mère. En revanche, pour son mari extérieur au groupe de matrilignage, celleci n'est qu'une épouse, elle est dissociée de la mère. Une femme n'a avec son mari que des rapports sexuels qui n'impliquent pas nécessairement la procréation. Les partenaires se choisissent plus librement et se quittent aussi plus librement parce que peu engagés dans la filiation. Les femmes y sont plus respectées et plus libres. Les mariages y sont très instables.

La fonction paternelle est différente dans chacun de ces systèmes.

Dans les sociétés matrilinéaires, co-existent deux fonctions paternelles. L'une, est détenue par le père, l'autre par l'oncle maternel. Les enfants appartiennent au lignage de leur mère, régi par les oncles maternels. Ces derniers sont les garants de l'appartenance au groupe. C'est un groupe unifié par un même lien au corps. L'oncle maternel a en charge la vie des enfants, il doit les nourrir, les soigner, les guérir. L'oncle maternel détient les terres, les magies, et les transmet à ses neveux selon son bon gré. Il peut dans certains cas exercer une loi arbitraire. Quant au père, c'est un étranger. Il reste extérieur au groupe de ses enfants. Il ne partage pas avec eux le même sang ni le même corps. Il n'est pas géniteur de ses enfants car il ne transmet aucune substance corporelle qui viendrait de lui ou de son lignage.

Dans les sociétés patrilinéaires, le père appartient au groupe de ses enfants. Père et enfants partagent le même sang, le même corps. Ils ont des substances corporelles communes. Le père doit assurer la survie de ses enfants, les nourrir, les soigner. Il peut sanctionner l'enfant s'il dévie. Il peut l'exclure. Il représente le groupe. Il est détenteur des deux formes d'autorités, celle conférée par la représentation des ancêtres et celle conférée par l'appartenance au même lignage que ses enfants.

Selon la filiation patrilinéaire, un père est autre en tant qu'il représente les membres de sa descendance, et les ancêtres. En revanche dans un système de parenté matrilinéaire, un père est altérité en tant qu'il appartient à un autre matrilignage que celui de ses enfants. Les fonctions paternelles ainsi définies par leur extériorité peuvent être mises en parallèle avec les objets de discours qui sous-tendent les croyances de la religion et de la magie.

La religion implique un corps d'intermédiaire et un groupe de fidèles, elle dispense des 
grâces. La magie fait appel à une puissance impersonnelle mise en œuvre dans des formules et des pratiques rituelles, elle est l'apanage d'individus isolés qui ne font pas église. Quant à la sorcellerie, elle entraîne des pratiques maléfiques ou des textes d'accusation. Les systèmes patrilinéaires sont étayés par la religion. Les systèmes matrilinéaires sont étayés par la magie.

L'objet sacré est un objet d'exception au cœur de la religion. Il est un objet du monde, sélectionné par un groupe pour représenter l'au-delà. Il est tabou et interdit. Il appartient au monde et il est inclus dans l'au-delà. Il représente autre chose que lui-même. De l'objet sacré on peut passer à l'objet sacrificiel. Ce dernier est un objet du monde dont on va détruire l'appartenance pour renforcer la représentation de l'autre monde. Tout le système religieux joue de cette disjonction accusée mais reliée de l'appartenance au monde d'ici et de la représentation de l'autre monde.

La fonction paternelle patrilinéaire est associée à l'objet sacré. Tout comme lui, dans les systèmes patrilinéaires, le père représente un au-delà tout en appartenant au monde réel. Il appartient au groupe de ses enfants, et il représente les ancêtres et tous ceux qui l'ont précédé. Un père héritier de ses ascendants, dit « moi » pour parler d'eux. Il est totalement identifié à eux. C'est parce qu'à la fois il représente ses ancêtres et appartient au lignage de ses propres enfants qu'il possède une autorité puissante.

Les objets magiques ont pour caractéristique d'être dépouillés de leur valeur d'usage et de leurs attributions courantes. À la différence de l'objet sacré qui est la propriété inaliénable d'un groupe, l'objet magique est la possession aliénable d'un individu. Il peut changer de main. Inlassablement obtenu et perdu, il est une source intarissable de drames inattendus. L'objet magique appartient à un au-delà non défini, un au-delà non structuré. Il est introduit dans le monde grâce à la formule magique. Puisque l'objet magique est un réel de l'audelà, il ne peut pas être sacrifié car la destruction de l'objet magique met fin à ses propriétés. La magie ne cherche pas à jouer sur la disjonction des deux mondes, mais au contraire, tente de les concilier. La magie efface les contradictions. Si la magie échoue, c'est que le rite n'a pas été bien réalisé, ou alors c'est qu'il y a une contre-magie. La force de l'objet magique tient non pas à une transcendance supérieure, mais à un au-delà impersonnel qui lui confère une origine opaque, voisine du monde obscur de la sorcellerie. L'appartenance de l'objet magique étant extérieure à son champ d'action, le discours de la magie ne connaît pas de sacrifice. L'objet magique a pour fonction d'inclure son détenteur dans les normes culturelles et usuelles de sa société.

La fonction paternelle matrilinéaire est associée à la magie. Le père appartient à un autre lignage que ses enfants, il ne peut agir sur eux. Il est relié à ses enfants par les représentations, l'apparence, et a pour fonction de les aider à s'intégrer dans le monde humain. Les « relations à plaisanterie », forme d'incroyance, de banalisation, servent à pacifier les conflits et à séculariser l'ordre établi.

Contrairement aux croyances de la religion et de la magie, la sorcellerie implique un audelà, un «monde de la nuit» dans lequel un sorcier est supposé entraîner sa victime afin qu'elle puisse y devenir sorcière à son tour. Aussi appartenance et inclusion sont-elles indéfiniment réversibles. La croyance en la sorcellerie est étroitement associée dans les sociétés traditionnelles à la relation duelle mère/enfant, à l'exclusion des tiers paternels.

Dans sa conclusion, l'A. se demande si un parallèle avec l'évolution de la paternité dans nos sociétés est possible? Tout comme dans les systèmes matrilinéaires, sexualité et procréation sont dissociées. Les pères ne peuvent plus ni exclure ni sanctionner durement. L'individualisme de notre société a ceci de commun avec la magie, qu'il ne fait pas église. Nous serions peut-être en train de glisser vers une fonction paternelle matrilinéaire.

Martine Gross.

124.46

ROALD (Anne-Sofie).

Women in Islam. The Western Experience. New York, Routletge, 2001, 339 p. (bibliogr., glossaire, index).

D'origine norvégienne, convertie à l'islam depuis 1982 et mariée à un musulman palestinien, A.-S.R. sait par expérience le poids qu'exercent la classe sociale, le genre, l'appartenance ethnique et nationale, la tradition religieuse et la condition minoritaire/majoritaire sur les relations que chacun entretient avec l'islam en termes identitaires comme dans le jeu de l'interaction sociale. Ayant conquis légitimité et écoute aussi bien auprès des musulmans que des Européens, elle utilise cette position biographique privilégiée pour observer comment la rencontre culturelle entre l'islam et l'Occident peut provoquer des changements dans l'actuelle législation islamique et entamer un processus d'innovation à l'encontre du statut des femmes. 